\title{
Bi-layer functionally gradient thick film semiconducting methane sensors
}

\author{
A BANERJEe, A K HALDAR, J MONDAL, A SEN* and H S MAITI \\ Electroceramics Section, Central Glass and Ceramic Research Institute, Kolkata 700 032, India
}

\begin{abstract}
Gas sensors based on metal oxide semiconductors like tin dioxide are widely used for the detection of toxic and combustible gases like carbon monoxide, methane and LPG. One of the problems of such sensors is their lack of sensitivity, which to some extent, can be circumvented by using different catalysts. However, highly reactive volatile organic compounds (VOC) coming from different industrial and domestic products (e.g. paints, lacquers, varnishes etc) can play havoc on such sensors and can give rise to false alarms. Any attempt to adsorb such VOCs (e.g. by using activated charcoal) results in sorption of the detecting gases (e.g. methane) too. To get round the problem, bi-layer sensors have been developed. Such tin oxide based functionally gradient bi-layer sensors have different compositions at the top and bottom layers. Here, instead of adsorbing the VOCs, they are allowed to interact and are consumed on the top layer of the sensors and a combustible gas like methane being less reactive, penetrates the top layer and interacts with the bottom layer. By modifying the chemical compositions of the top and bottom layers and by designing the electrode-lead wire arrangement properly, the top layer can be kept electrically shunted from the bottom layer and the electrical signal generated at the bottom layer from the combustible gas is collected. Such functionally gradient sensors, being very reliable, can find applications in domestic, industrial and strategic sectors.
\end{abstract}

Keywords. Sensor; semiconductor; tin dioxide; functionally gradient.

\section{Introduction}

Gas sensors using metal oxide semiconductors were first proposed by Seiyama et al (1962) and Taguchi (1962). The recent concern over environmental pollution and efficiency in a variety of combustion processes and of increased awareness over the need to monitor potentially hazardous gases have stimulated substantial research and development in the field of gas sensors. Such sensors are primarily based on $n$-type semiconductors like $\mathrm{SnO}_{2}$, where loss of oxygen on heating results in the generation of oxygen vacancies (balanced by the reduction of some of the $\mathrm{Sn}^{4+}$ ions to $\mathrm{Sn}^{2+}$ ions) leading to electron donor states. In the first reaction, atmospheric oxygen becomes chemisorbed to the surface, consuming the electron as given below

$$
\mathrm{O}_{2}+2 e^{-} \rightarrow 2 \mathrm{O}_{\text {ads }}^{-} \text {. }
$$

As the free electrons are consumed, the conductivity decreases. Any reducing gas, present in the ambient, produces a counter reaction where, the reducing gas removes the chemisorbed oxygen, frees the bound electrons and increases the conductivity of the semiconducting gas sensors thus generating a signal. However, such sensors respond to different reducing gases and to impart selectivity, normally the following three methods (Roy Morrison

\footnotetext{
*Author for correspondence
}

1987) are tried. They are (i) the use of catalysts and promoters, (ii) modification of the operating temperature and (iii) the use of filters. Normally palladium is used as a catalyst (Kohl 1990; Gopal and Schierbaum 1995) and is an indispensable component in $\mathrm{SnO}_{2}$ sensors, especially for the oxidation of hydrocarbons. Incidentally, highly reactive volatile organic compounds (VOC) emanating from different industrial and domestic products like paints, lacquers, varnishes can strongly influence (SchweizerBerberich 1999) such sensors and can give rise to false alarms even in the absence of detecting gases like methane. These VOCs are highly reactive and so far, two techniques have been attempted to circumvent the problem. One is the use of charcoal filter (Schweizer-Berberich et al 1999). The disadvantage of this method lies in the fact that charcoal filters also adsorb most of the combustible gases and hence cannot be used to detect satisfactorily the combustible gases like methane, propane, LPG etc in presence of VOCs. The other alternative (Papadopoulos et al 1996) is to use uncoated/coated ( $\mathrm{Pt}, \mathrm{Pd})$ filters of $\mathrm{Al}_{2} \mathrm{O}_{3}, \mathrm{SiO}_{2}, \mathrm{WO}_{3}$ etc on $\mathrm{SnO}_{2}$. However, the second method gives protection primarily against alcohol crosssensitivity, whereas VOCs, in general, are either not affected by such filters, or the overall sensitivity of the sensors dramatically goes down.

In the present work, a novel bi-layer functionally gradient sensor has been developed to get round the problem. Here, instead of adsorbing the VOCs, they are allowed to interact and are consumed on the top layer of the sensors 
and the combustible gases like LPG, methane being less reactive penetrate the porous top layer and interact with the bottom layer to generate the desired signal.

\section{Experimental}

Batches containing tin dioxide and antimony (0-1 wt.\% as $\left.\mathrm{Sb}_{2} \mathrm{O}_{3}\right)$; tin dioxide, palladium $\left(0-2 \mathrm{wt} . \%\right.$ as $\left.\mathrm{Pd}^{0}\right)$ and antimony (0-1 wt.\% as $\mathrm{Sb}_{2} \mathrm{O}_{3}$ ) were prepared by simultaneous precipitation technique. In this technique, stannous chloride $\left(\mathrm{SnCl}_{2}, 2 \mathrm{H}_{2} \mathrm{O}\right)$ solution was made in water containing a very small amount of hydrochloric acid (1-2\%). Antimony oxide $\left(\mathrm{Sb}_{2} \mathrm{O}_{3}\right)$ was dissolved as antimony chloride in hydrochloric acid $(50 \%)$ and $\mathrm{PdCl}_{2}$ was dissolved as $\mathrm{Pd}\left(\mathrm{NO}_{3}\right)_{2}$ in hot nitric acid $(\sim 20 \%)$. The above solutions were added to ammonium hydroxide solution ( $\mathrm{pH}$ above 10 and under stirring) so that all the hydroxides were precipitated simultaneously. The precipitates were washed, made chloride free and finally calcined at $900^{\circ} \mathrm{C}$ $(2 \mathrm{~h})$ to get the oxide powders. The phase identification of the calcined powders was carried out by X-ray diffraction (Philips, PW 1710). Particle morphology of the powders was observed in a scanning electron microscope (Leo, 30i).

Thick pastes of the powders were prepared in an aqueous medium containing a small amount of ethyl cellulose binder. The pastes were painted on the outer surface of thin alumina tubes (length $3 \mathrm{~mm}$, outer diameter $2 \mathrm{~mm}$ and thickness $0.5 \mathrm{~mm}$ ) with gold electrode and platinum lead wires already attached at the ends (by curing at a high temperature). Kanthal heating coils were placed inside the tubes. The consistency of the pastes and the processing variables were optimized to get final coatings of $50-60 \mu \mathrm{m}$ thickness. For the functionally gradient bi-layer sensors the bottom layer was a coating of a powder containing tin dioxide, antimony oxide and palladium, whereas the top layer was a coating of tin dioxide, palladium and alumina powder (5-30 wt.\%). After applying the bottom coating, the assembly was dried in an oven followed by application of the top coating. Finally, the coated alumina tubes were cured at a temperature in the range of $600-1000^{\circ} \mathrm{C}$ for $3 \mathrm{~h}$. The electrical resistance and methane $(500 \mathrm{ppm})$ sensitivity of the coatings were measured at different temperatures (up to $350^{\circ} \mathrm{C}$ ) by using a digital multimeter (Solartron), a constant voltage/current source (Keithley 228A) and $\mathrm{X}-\mathrm{Y}$ recorder (Yokogawa). All the fired samples were initially preheated at $350^{\circ} \mathrm{C}$ for $72 \mathrm{~h}$ to achieve the desired stability before the measurements.

\section{Results and discussion}

The X-ray diffractogram of calcined tin hydroxide powder is depicted in figure 1 , which indicates complete $\mathrm{SnO}_{2}$ phase formation after firing at $900^{\circ} \mathrm{C}$. The scanning elec- tron micrograph of the calcined powders (figure 2) shows particles of the order of $100 \mathrm{~nm}$ size. The typical trend of resistance vs temperature (figure 3) of pure $\mathrm{SnO}_{2}$ can be understood by considering the competitive adsorption of oxygen and moisture on $\mathrm{SnO}_{2}$ surface from the ambient (Saha et al 2001). Incidentally, antimony oxide is often added (Potthast and Schumann 1999) to tin dioxide in real life sensors to enhance their conductivity. On doping with antimony, $\mathrm{SnO}_{2}$ shows a decrease in resistivity owing to substitution of $\mathrm{Sn}^{4+}$ by $\mathrm{Sb}^{5+}\left(\mathrm{Sb}^{5+}\right.$ should be predominant at the firing temperature) leading to an increase in carrier concentration (donor doping) (Rajpure et al 2000). Hence Sb-doped samples show orders of magnitude lower resistance than that of pure $\mathrm{SnO}_{2}$. The effect of Sb-doping on the sensitivity $(S)$ of $\mathrm{SnO}_{2}$ samples in $500 \mathrm{ppm}$ methane at $350^{\circ} \mathrm{C}$ has been depicted in figure 4 where the sensitivity $(S)$ is given by

$$
S=\left(R_{\mathrm{A}}-R_{\mathrm{G}}\right) / R_{\mathrm{A}} \times 100 \%,
$$

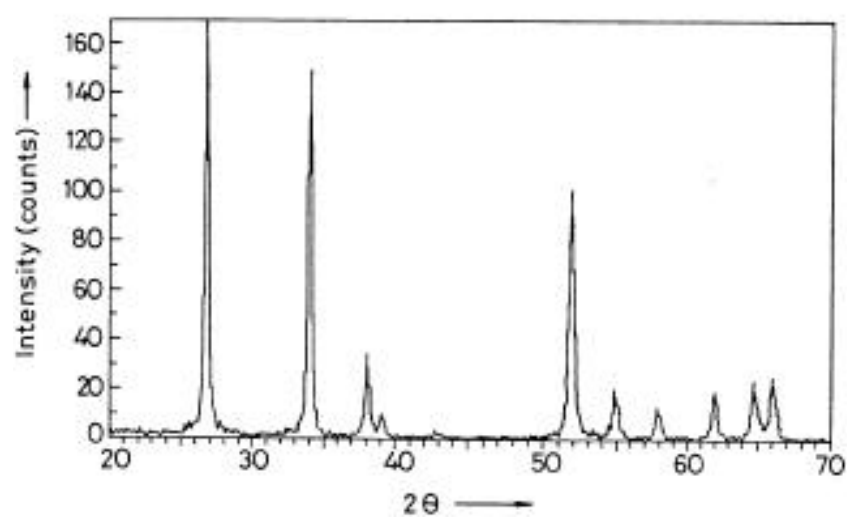

Figure 1. X-ray diffractogram of precipitated tin hydroxide powder after calcination at $900^{\circ} \mathrm{C}$.

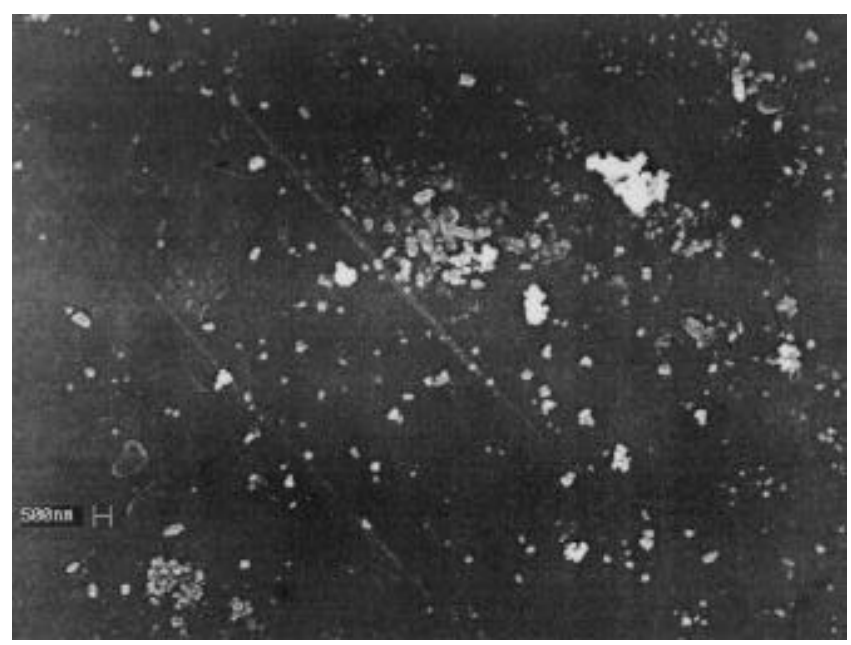

Figure 2. Scanning electron micrograph of a typical calcined powder. 


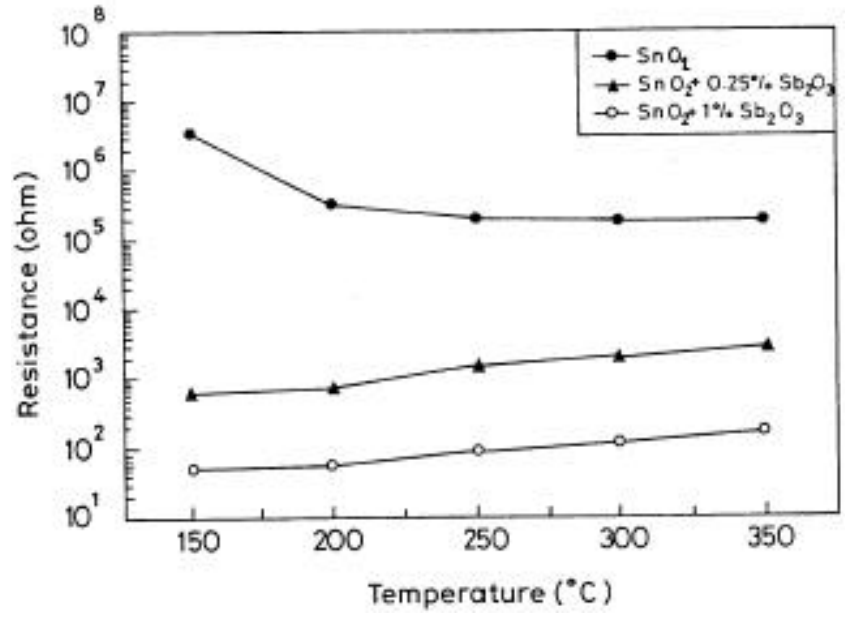

Figure 3. Temperature dependence of resistance of tin dioxide coatings having different amounts of antimony doping.

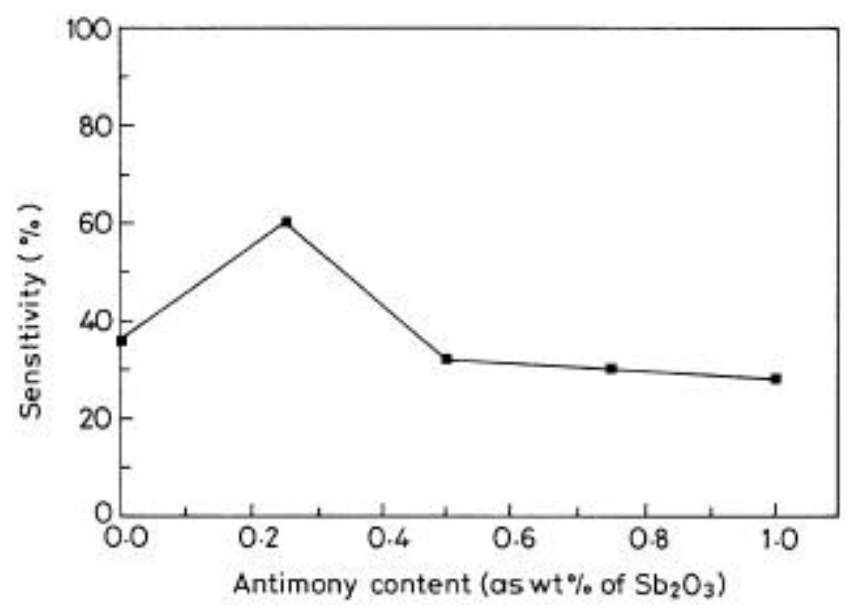

Figure 4. Effect of antimony doping on methane sensitivity of tin dioxide coatings.

$R_{\mathrm{A}}$ and $R_{\mathrm{G}}$ being the sensor resistance in air and gas at the same temperature. The measurement temperature, $350^{\circ} \mathrm{C}$, was selected for our studies because $\mathrm{CH}_{4}$ sensitivity is maximum around this temperature. As discussed earlier, $\mathrm{Sb}^{5+}$, being donor dopant, increases the carrier concentration of $\mathrm{SnO}_{2}$ samples and hence improve the sensitivity at $0.25 \%$ doping level. Higher doping level lowers the sensitivity as shown in figure 4 . Such high-antimony doped samples, whose resistance is already low (even with chemisorbed oxygen on their surfaces in ambient), are not expected to show much lowering in resistance in presence of reducing gases like methane and hence their sensitivity values should be poor as corroborated by our observation (figure 4).

The top layer of the functionally gradient bi-layer sensor contains tin dioxide, palladium and alumina. Alumina raises the resistance of the top coating to the order of $10-100 \mathrm{M} \Omega$ at the operating temperature. Such increase in resistance can be explained by considering the electronic interaction between semiconducting tin dioxide grains in close contact with the Lewis acid sites (electron acceptor) of alumina grains (formed during heating, see (3)).

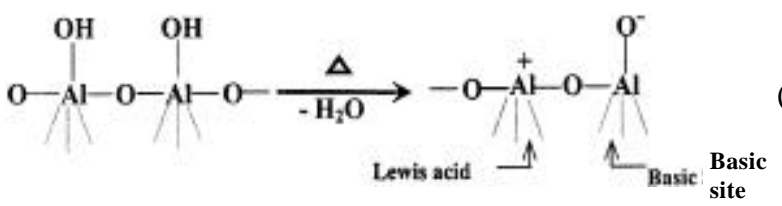

Whereas, the bottom coating is devoid of alumina and contains antimony resulting in the resistance value of the order of $10-100 \mathrm{k} \Omega$ at the operating temperature. The VOCs being highly reactive, interact with adsorbed oxygen on the top layer releasing free electrons. However, due to three orders of higher resistance of the top coating with respect to that of the bottom coating, the top coating always remains shunted to the bottom coating. Less reactive gases like methane penetrate the top layer and interacts with the bottom layer of adsorbed oxygen and the change in resistance is picked up by the electrical leads at the bottom. Such functionally gradient bi-layer sensors can show a sensitivity in the range of $70-90 \%$ in methane and $20-40 \%$ in the presence of VOCs, whereas standard single coated sensors show a sensitivity of $80-95 \%$ in the presence of VOCs under identical conditions.

\section{Conclusions}

By modifying the chemical compositions of the top and bottom layers, novel functionally gradient bi-layer tin dioxide based sensors have been developed, which show excellent sensitivity towards methane with negligible cross-sensitivity towards volatile organic compounds.

\section{References}

Gopal W and Schierbaum K D 1995 Sensors and Actuators B26-27 1

Kohl D 1990 Sensors and Actuators B1 158

Papadopoulos C A, Vlachos D S and Avritsotis J N 1996 Sensors and Actuators B32 61

Potthast H and Schumann B 1999 U.S. Patent 5,942,679

Rajpure K Y, Kusumade M N, Neuman-Spallart M N and Bosale C H 2000 Mater. Chem. and Phys. 6484

Roy Morrison S 1987 Sensors and Actuators 12425

Saha M, Banerjee A, Halder A K, Mondal J, Sen A and Maiti H S 2001 Sensors and Actuators B79 187

Schweizer-Berberich M, Strathmann S, Weimar U, Sharma R, Seube A, Peyre-Lavigne A and Gopel W 1999 Sensors and Actuators $\mathbf{B 5 8} 318$

Seiyama T, Kato A, Fujiishi K and Nagatani M 1962 Anal. Chem. 341502

Taguchi N 1962 Japan Patent 45-38200 